\title{
Indices
}

\section{Index of Persons}

Ablabius 37, 38, 39, 124

Agbenuti H. 126

Alès A. de 102, 126

Alexandre M. 126

Alszeghy Z. 5, 6, 128

Altaner B. 126

Altenburger M. 126

Ambrose VI, 6, 7, 8, 90, 95, 100, 103, 104-111, $122,123,130,131$

Ambrosiaster VI, 7, 8, 79, 114-118, 122, 123, 129, 132

Amo Usanos R. 126

Andersen C. 126

Andia Y. de 17, 23, 126

Andrzejewski R. 126

Armitage Robinson J. 15, 17, 21, 24, 125

Armstrong A.H. 42, 126

Armstrong G.T. 14, 126

Athanasius 6, 7, 28, 130

Augustine VI, VIII, 1, 3-9, 59, 77, 79-99, 111, 117, $121-124,127-129$

Badurina T. 31, 32, 126

Balás D.L. 6, 42, 56, 122, 126

Balthasar H.U. von 42, 127

Bammel C.P. 78, 127

Bardy G. 127

Barkley G.W. 63, 68, 69, 126

Basil 6, 7, 42, 126

Bastero J.L. 124

Baudry G.H. 127

Beatrice P.F. 76, 77, 127

Belindo C. 127

Bennet B. 77, 127

Benoît A. 11, 33, 127

Bianchi V. 127

Blic J. de 80, 81, 91, 127

Bogan M.I. 92, 124

Bonwetsch G.N. 29, 36, 127

Bouchet J.R. 127

Boulding M. 81-83, 124

Boyer Ch. 127

Bracht K. 31, 32, 36, 127

Brawne C.G. 118, 124,

Bray G.L. 114-117, 123
Buonaiuti E. 127

Butterworth G.W. 60, 126

Cassel J.D. 127

Casteluccio G. 127

Chadwick H. 64, 66, 69, 78, 126, 127

Cherniss H.F. 41, 127

Clémence J. 127

Cleveland Coxe A. VII

Crouzel H. 59, 74, 127

Cwyl K. 10

Cyprian 7

Cyril of Jerusalem 7

Daniélou J. 127, 128

Dekert T. 17, 33, 128

Desalvo C. 128

Deutsch S. 128

Didier J.C. 128

Didymus the Blind VI, 8, 10, 77, 112-113, 122, $124,127,129$

Donaldson J. VII

Douglass S. 124

Drobner H.R. 125

Dubarle A.M. 128

Dyson R.W. 86, 123

Echari M.A. 128

Eunomius 37, 39-42, 46, 48, 124, 126

Farges J. 128

Ferguson E. 57, 125, 128

Fernandez J.M. 128

Filipowicz A.M. 128

Fitzgerald A. 128

Flick M. 5, 6, 128

Floëri F.R. 128

Francesconi G. 128

Gaith J. 53, 55, 128

Gasparo G.S. 75, 128

Gaudel A. 28, 76, 77, 87, 107, 108, 114, 128

Gonzáles S. 128

Graef H.C. 51-53, 125

Granat W. 4, 128

Grant R. 128

Gregory of Nyssa V, VIII, 1, 6-8, 10, 11, 28, 33, 37-58, 62, 114, 120, 121, 124-131

Gregory of Nazianzus VI, 9, 118-120, 122, 124, 132

Grelot P. 128 
Grodecki T. 42

Gross J. 5, 10, 28, 30, 53, 55, 68, 75, 80, 87, 91, $96,111,113,114,117,120,128$

Haag H. 128

Hadot P. 128

Hall S.G. 46, 48, 54, 124, 125

Hamman A.G. 129

Hammond Taylor J. 81, 124

Heine R.E. 47, 55, 61, 63, 67, 125, 126

Hilary of Poitiers 7

Hill E. 97, 99, 124

Hooker M. 66, 126

Huhn J. 106, 109, 129

lamblichus 42

Irenaeus V, 2, 7-37, 55, 62, 68, 75, 76, 80, 100, $102,107,113,114,121,125,126-132$

Ivanka E. 129

Jacob C. 129

Jaeger W. VII

Jäntsch J. 117, 118, 129

Julian of Eclanum VIII, 4, 84, 85, 91, 92, 9-96, 117,123

Kannengiesser Ch. 129

Karfiková L. 124

Kelly J.N.D. 6, 7, 27, 33, 101, 102, 129

Kochańczyk-Bonińska K. 10

Lactantius 7

Lafont G. 129

Lamirande E. 129

Langemeyer G. 4, 129

Langstadt E. 129

Laporte J.P. 3, 74, 75, 129

Lassiat H. 12, 17-19, 21, 26, 28, 29, 65, 129

Layton R.A. 129

Leeming B. 79, 129

Leipoldt J. 129

Leśniewski K. 33, 129

Leys R. 129

Libera P. 129

Lienhard J.T. 68, 126

Loiselle A. 111, 129

Lyonnet S. 5, 6, 87, 129

Madec G. 129

Magne J. 129

Malherbe A.J. 57, 125

Manikowski M. 41, 130

Mann F. 126

Mara J. 107, 109, 111, 130

Mara M.G. 130

Marcinkowska M. 10
Mateo-Seco L.F. 124

McCambley C. 54, 125

McClear E. 53, 56, 121, 130

McHugh M.P. 105, 123

Mejzner M. 30, 130

Ménard J.E. 83, 102, 130

Merlin N. 130

Methodius of Olympus V, 7-9, 11, 29-37, 62, 121, $125,127,128,130$

Migne J.P. VII

Milko P. 130

Moriarty R. 48, 125

Musurillo H. 33-37, 125

Myszor W. 130

Nakonieczny R. 130

Naumowicz J. 10

Ní Riain Í.M. 105, 106, 108, 109, 111, 123

Nielsen J.T. 130

Norris R.A. 55, 125

Orbe A. 10, 11, 16, 21-23, 26, 27, 62, 63, 68, 130

Origen V, 3, 7-9, 29, 37, 43, 59-79, 102, 106, 114 , $120,121,125-132$

Otis B. $6,29,32,33,130$

Pałucki J. 130

Paredi A. 130

Patterson L.G. 29, 36, 37, 130

Pelagius 91, 118, 123, 129

Pépin J. 130

Peretto E. 130

Philo of Alexandria 3, 25, 27, 28, 74, 75, 103, 127,129

Pietras H. 130, 132

Pisi P. 75, 130

Plato 29, 64, 77, 129

Plezia M. 8, 130

Plotinus 42, 77

Pochoshajew I. 124

Porphyry 42

Potrzebnicka E. 10

Prinzivalli E. 130

Proclus 42

Przyszychowska M. I, III, VI, VIII, IX, 43, 57, 130, 131

Quasten J. 131

Ramsey B. 87-90, 124

Reale G. 43, 131

Régis B. 131

Rivière $J .131$

Roberts A. VII

Romer J. 131 
Rondet H. 13, 79, 131

Roth C.P. 46, 58, 125

Rufinus 8

Sage A. 131

Salij J. IX, 1

Savage J.J. 104, 123

Savon H. 131

Schaff P. VII, 44

Scharbert J. 131

Scharl E. 33, 131

Scheck Th.P. 65, 70-74, 126

Schutz D.R. 131

Scheffczyk L. 2, 3, 5, 15, 18, 19, 27, 29, 30, 32, $34,56,69,70,75,76,78,79,87,91,100$, 101, 105, 106, 110, 111, 113, 117, 120, 131

Scognamiglio R. 131

Simonetti M. 64, 65, 74, 131

Simplician 88-90, 123, 124

Slomkowski A. 131

Spiechowicz-Kristensen G. 10

Springer T. 131

Stanula E. 117, 131

Stead Ch. 43, 131

Steenberg M.C. 131

Stępień T. 10

Straffner H. 131

Stramara D. 39, 40, 124

Strohm M. 131

Swallow J.E. 118, 124

Szabó F. 103, 131

Szczerba W. 36, 131

Szewczyk P.M. 132

Szram M. 75, 76, 132

Szymusiak J.M. 119, 120, 132

Teitchtweiter G. 75, 132

Tennant F.R. 4, 6, 13, 27, 28, 32, 59, 67, 70, 77, 111, 132

Tertullian VI, 7, 8, 100-103, 122, 126, 129, 131

Teske R.J. 83-86, 91, 92, 94-98, 123

Testa E. 5, 132

Thomas Aquinas IX,

Torano Lopez E. 132

Tum D. 132

Turmel J. 76, 106, 132

Valentinus 25

Valero J.B. 114-116, 118, 132

Vanneste A. 5, 132

Verna Harrison N. 118, 119, 124

Verriele A. 11, 132

Vicario L. 132
Viciano A. 125

Vinson M. 119, 124

Vives J. 10, 13, 14, 16, 33, 48, 55, 56, 121, 132

Vöglte A. 132

Wace T. VII

Wagner H. 4, 132

Wencel K. 132

Wierzbicka J. 10

Wiley T. 5, 132

Williams N.P. 4, 7, 59, 61, 77, 78, 80, 93, 98, 102, 107, 120, 132

Williams R. 78, 127

Woods Callahan V. 50, 124

Wójcik A. 10

Zachhuber J. 124

Zarzeczny R. 10

\section{Index of Biblical References}

Gen 1:26 15, 101
Gen 1: 2744
Gen 1:28 101
Gen 2:7 18, 90
Gen 2:16-17 104, 131
Gen 2:17 12, 31, 49, 81
Gen 3:8 20
Gen 3:14 105
Gen 3:17 66
Gen 3:19 36
Gen 3:21 46, 63, 127
Gen 3:22 66
Gen 12:3 85
Ex 20:26 63
Lev 12:8 70
Lev 21:11 69
Jb 14:4 68, 69
Jb 14:4-5 68
Ps 21:16 73
Ps 50:5 110
Ps 50:7 68-70, 85
Ps 50:9 110
Ps 50:11 110
Ps 50:14 110
Ps 57:4 69
Ps 81:6 66
Ps 81:7 66
Ps 110:4 95
Sir 33:10-15 89


Sir 33:11 89

Wis 2:24 97

Wis 2:24-25 97

Is 50:1 115

Jer 18:3-4 35

Ezk 18:4 73

Ezk 37:7-8 62

Mt 6:12 20

Mt 9:2 20

Mt 18:12 48

Mt 18:12-13 48, 61

Mt 18:12-14 132

Lk 1:78 20

Lk 15:1-7 46

Lk 15:4-5 61

Lk 15:4-6 18

Lk 19:10 108

Jn 1:13 VIII

In 1:16 112

Jn 3:5 68

In 9:3 18

In 9:6 18

In 9:7 26

Rom 5:12 83, 86, 92, 96-98, 114, 116

Rom 5:12-14 129

Rom 5:12-21 5, 6, 128, 129

Rom 5:14 64, 71

Rom 5:17 73, 74

Rom 5:18 72

Rom 7:9 30

Rom 9:21 87, 88

Rom 9:24 89

Rom 11:16 88, 115

Rom 14:9 108

1Cor 1:28 108

1Cor 15:22 51, 64, 88

1Cor 15:45 67

1Cor 15:49 103

1 Cor 15:53 57

2Cor 5:4 63

Ga 3:27 112

Eph 4:26-28 81

Php 2:8 13

1Tim 2:5 20

Hbr 7:9-10 8 